\title{
A SIMPLE ALGORITHM TO DETECT BALANCE IN SIGNED GRAPHS *
}

\author{
Frank HARARY and Jerald A. KABELL \\ Institure for Social Research, University of Michigan. Ann .4rbor, MI, U.S.A.
}

Communicuted by K.H. Kim

Received 16 November 1979

Dedicated to Dorwin Cartwright on his 65 th birthday

\begin{abstract}
We develop a natural correspondence between marked graphs and balanced signed graphs, and exploit it to obtain a simple linear time algorithm by which any signed graph may be tested for balance.
\end{abstract}

Keywords: Signed graph, Algorithm, Balance spanning tree, Marked graph.

\section{Introduction}

Psychologists and sociologists have long been ac justomed to using signed graphs to model group structure in cases where the relations.rips between entities can, in some sense, be classified as 'positive' and 'negative,' i.e., love- hate, ally-enemy', etc. The concept of balance in such a situation first explicitly appears in Heider (1946) and was independently rediscovered by Harary (1953-54). It has since been extensively developed by Cartwright and Harary (1956) and Harary (1959).

Throughout our discussion, we shall be using the graph theoretic notation and terminology of the book (Harary (1969)). For completeness we recount here the definitions of the concepts central to this theory. A signed graph is a graph whose lines have all been designated either positive or negative. The sign of a cycle in a signed graph is simply the product of the signs of all the lines of the cycle. A signed graph is said to be balanced if every cycle in it is postive. A spanning tree of a connected graph $G$ is any subgraph of $G$ which is maximal with respect to the constraint that it contains no cycles. Thus a spanning tree of $\boldsymbol{G}$ is a subtree which contains all the points of $\boldsymbol{G}$.

The essential structural feature of a balanced signed graph is given in the following theorem of Harary (1953-54).

- Research supported in part by NSF grant SOC 76-16824. 


\subsection{Structure theorem for balance}

A signed graph $S$ is balanced if and only if its points can be partitioned into two sets. called coalitions, in such a way that each positive line joins two points of the same coalition while each negative line joins two points in different coalitions.

In a marked graph, the points are designated positive or negative in contrast 10 a signed graph in which the lines carry the signs. The large scale application of the theory of balanced signed graphs can be facilitated by providing an efficient procedure for testing a given signed graph for balance. By exploiting a natural correspondence between balanced signed graphs and marked graphs, we are now able to devise a very simple linear-time algorithm for carrying out this testing.

\section{The correspondence between marked graphs and balanced signed graphs}

In social psychological terms, one might think of the positive points of a marked graph as truthful persons and the negative points as inveterate liars. Extending this line of thought, one is naturally interested in those marked graphs in which a person who starts a rumor will always hear it return to him in its original form. More precisely, a consistent marked graph is one in which the product of ihe signs of the points in anj cycle is positive. $T_{1}$ us a consistent marked graph is the analogue of a balanced signed graph. Beineke and Harary (1978b) made the first extensive study of marked graphs and also of marked digraphs (Beineke and Harary (1978a)) and were able to obtain a number of necessary conditions for a marked graph to be consistent.

Let $M$ be a marked graph with underlying graph $G=G(M)$ having the same points and lines as $M$, but without any signs on its lines or points. The signed graph of the marked graph $M$, written $S(M)$, is obtained from $G$ by affixing to each line the product of the signs of its two points. It is easy to see that for every marked graph $M$ (whether consistent or not), the signed graph $S(M)$ is balanced.

The natural next question is whether every balanced signed graph can be obtained in this fashion from a marked graph. The somewhat surprising answer is that it can indeed; in fact, each balanced signed graph can be realized this way from precisely two (possibly isomorphic) marked graphs, each of which can be obtained from the other by reversing the signs on the points. We wish to thank Lászlo Babai for providing the essential idea in the proof of this last statement.

We now formalize the foregoing discussion in the following theorem, first presented in Harary and Kabell (1979), whose proof yields the algorithm which will be presented in more detail in the next section. In the proof it is convenient to write $s(x)=+1$ or -1 for the sign of the point or line $x$. As a signed graph is balanced if and only if every component is balanced, there is no loss of generality in restricting our consideration to connected graphs.

Theorem 1 (Correspondence Theorem). To each connected marked graph $M$ there corresponds a unique balanced signed graph $B=S(M)$. To each conmected $B$ there correspond two marked graphs $M$ and $M^{\prime}$, which are sign-reversals, such that $S(M)=S\left(M^{\prime}\right)=B$. 
Proof. Let $M$ be a marked graph and let $B=S(M)$ be its signed graph. We now show that $B$ is balanced. Let $C=v_{1} e_{1} v_{2} c_{2} \cdots v_{m} c^{\prime} m v_{1}$ se a cycle in $B$. Then $s(C)=s\left(e_{1}\right) \cdot s\left(e_{2}\right) \cdots s\left(c_{m}\right)=$ $s\left(v_{1}\right) \cdot s\left(v_{2}\right) \cdot s\left(v_{2}\right) \cdots s\left(v_{m}\right) \cdot s\left(v_{m}\right) \cdot s\left(v_{1}\right)$, so $s(C)=\left(s\left(v_{1}\right)\right)^{2}\left(s\left(v_{2}\right)\right)^{2} \cdots\left(s\left(v_{m}\right)\right)^{2}=+1$.

Thus every cycle in $B$ is positive, hence $B$ is balanced.

Conversely, suppose $B$ is a balanced signed graph. Take $T$ to be any spanning tree in $B$, rooted at some point $v_{0}$. Assign $s\left(v_{0}\right)$ arbit rarily, suy positive. Now let $v_{1}, v_{2}, \ldots, v_{r}$ be the neighbors of $v_{0}$ in $T_{\text {, and }} e_{i}=v_{0} v_{i}, 1 \leqslant i \leqslant r$. For eacti of these, we set $s\left(v_{i}\right)=s\left(v_{0}\right) \cdot s\left(e_{i}\right)$. Multiplying by $s\left(v_{0}\right)$ shows that $s\left(e_{i}\right)=s\left(v_{0}\right) \cdot s\left(v_{i}\right)$. Using the same scheme, we can determine the sign of any point adjacent in $T$ to a previously signed point so that the sign on the line of $T$ joining them is the product of the signs on the two points, and thus all the points of $B$ can be given signs compatible with the signs of the lines of $T$. Now consider any chord $c$ of $T$, i.e., a line of $B$ not in $T$. The chord $e$ together with $T$ deternines a single cycle consisting of $e=v_{1} v_{k+1}$, and the unique path in $T$, written $v_{1} e_{1} v_{2} e_{2} \cdots v_{k} e_{k} v_{k+1}$, joining the two points of $e$. Since $B$ is balanced, the sign of this cycle, $s(e) \cdot s\left(e_{1}\right) \cdot s\left(e_{2}\right)$ $\cdots s(* k)$, must be positive, so $s(e)=s\left(e_{1}\right) \cdot s\left(e_{2}\right) \cdots s\left(e_{k}\right)$. In terms of the signs of points, however, $s\left(c_{1}\right) \cdot s\left(e_{2}\right) \cdots s\left(e_{k}\right)=s\left(v_{1}\right) \cdot s\left(v_{2}\right) \cdot s\left(v_{2}\right) \cdots s\left(v_{k}\right) \cdot s\left(v_{k}\right) \cdot s\left(v_{k+1}\right)=$ $s\left(v_{1}\right) \cdot\left(s\left(v_{2}\right)\right)^{2} \cdots\left(s\left(v_{k}\right)\right)^{2} \cdot s\left(v_{k+1}\right)=s\left(v_{1}\right) \cdot s\left(v_{k+1}\right)$ so $s(e)=s\left(v_{1}\right) \cdot s\left(v_{k+1}\right)$ and the sign of $e$ is compatible with the signs of the points generated by using $T$. Consequently the marked graph $M$ obtained by disregarding the signs on all the lines is one to which $B$ naturally corresponds, in the sense of the first part of the theorem.

Since the root of $T$ was signed positive arbitrarily. it is clear that the above argument can be carried through in a similar fashion for the cpposite choice of sign, negative, resulting in a marked graph $\boldsymbol{M}^{\prime}$, differing from $\boldsymbol{M}$ only ir that all point signs are reversed. Again $B$ naturally corresponds to $\boldsymbol{M}^{\prime}$. That $\boldsymbol{M}$ and $\boldsymbol{M}^{\prime}$ are the only two marked graphs to which $B$ corresponds is also evident, since each was complately determined by the choice of the root sign. We note that $M$ and $M^{\prime}$ might be isomorphic.

\section{Algorithm for testing balance}

The pioof of the Correspondence Theorem gives us the means to construct from a given balanced signed graph the two marked graphs to which it corresponds. It is equally evident that this construction yields a marking of the points of the graph which is compatible with the signing of the lines if and only if the signed graph is balanced. Hence. this gives a way in which any signed graph may be tested for balance. which we formalize as foll Jws.

Balance Algorithm. Given a signed graph $S$.

Step 1. Select a spanning tree $T$.

Step 2. Root $T$ at an arbitrary point, say $v_{0}$.

Step 3. Mark $v_{0}$ positive.

Step 4. Select an unsigned point adjacent in $T$ to a signed point.

Step 5. Label the selected point with the product of the sign of the previously signed 
point to which it is adjacent in $T$ and the sign of the line joining them.

Step 6. Are there any remaining unsigned points?

$$
\begin{aligned}
& \text { Yes - go to Step } 4 \\
& \text { No - go to Step } 7
\end{aligned}
$$

Step 7. Is there a line of $S-E(T)$ which has not been tested?

$$
\begin{aligned}
& \text { Yes - go to Step } 8 \\
& \text { vo - go to Step } 11
\end{aligned}
$$

Step 8. Select an untested line of $S-E(T)$.

Step 9. Is the sign of the selected line equal to the product of the signs of its two points?

$$
\begin{aligned}
& \text { Yes - go to Step } 7 \\
& \text { No - go to step } 10
\end{aligned}
$$

Step 10. Stop, $S$ is not balanced.

Step 11. Stop, $S$ is balanced.

This algorithm is quite convenient for testing a given signed graph for balance. We illustrate its use with the following example. Let $S$ be the signed graph shown in Fig. 1. Of course we see at once that $S$ is not balanced as it contains a negative triangle, $v_{1} v_{2} v_{3}$.

Following the first three steps of the algorithm, we select a spanning tree, root it, say at poin. $v_{2}$, and sign the root arbitrarily, as in Fig. 2 . The point signing procedure given in Steps 4-6 extends this to the signed and marked tree of Fig. 3.

The remainder of the algorithm then tests each of the remaining lines of $S\left(v_{1} v_{2}, v_{2} v_{4}\right.$, and $v_{4} v_{6}$ ) to determine whether their signs are compatible with the signs of their end points. In this example, the line $v_{1} v_{2}$ clearly results in $N o$ at Step 9 , causing the algorithm to terminate and to signal that $S$ is not balanced. If on the other hand we try the same example with the sign of $v_{1} v_{2}$ changed to negative, the algorithm will examine all of the remaining lines and discovering them compatible will terminate and signal that $S$ is balanced.

The two primary practical considerations with regard to any algorithm are the questions of implementation and complexity. In the case of the algorithm presented here, neither of these poses any difficulty. Tnere are available ir. ie literature (see, for example, Aho, Hopcroft and Ullman (1974)) several easily implemented linear time algorithms for

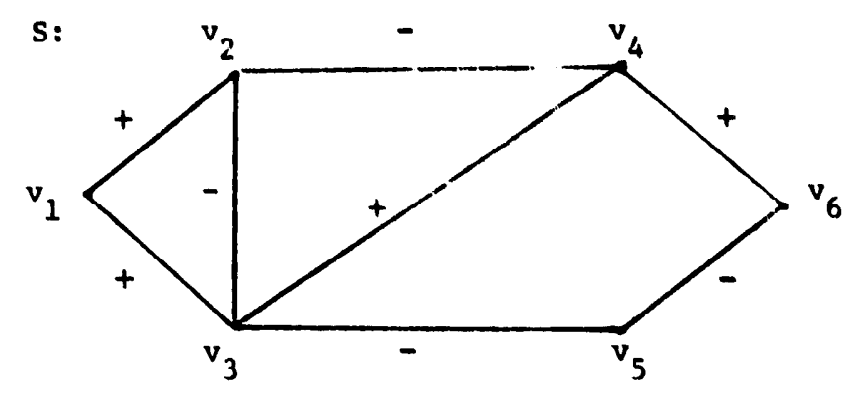

Fig. 1. 


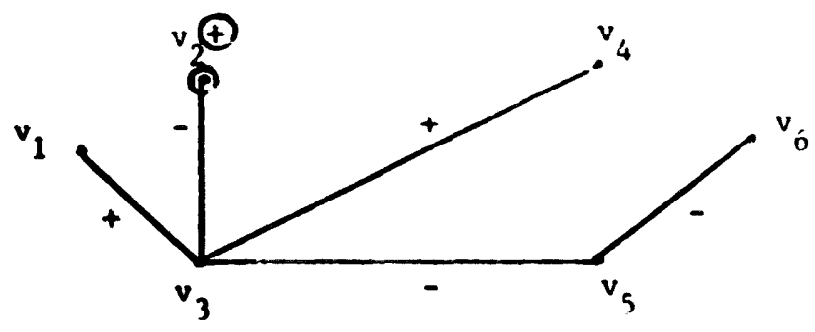

Fig. 2.

finding a spanning tree in a given graph. The remainder of our algorithm can clearly be programmed with minimal difficulty in any suitable high-level language. Since it requires sampling each line of the graph to be tested at most twice after the spanning tree has been found, the algorithm is certainly linear.

\section{Comments and further questions}

In Harary (1959) the point index and line index of balance of a signed graph $S$ are defined as the minimum number of points or lines which must be deleted from $S$ to obtain a balanced signed graph. The line index is of part cular interest since it is always the same as the minimum number of lines whose signs $m$ 'st be changed to yield a balanced signed graph. Exploiting the construction of Theorerı 1, we can obtain the following useful result.

Theorem 2. The line index of a signed graph $S$ is givin by the minimum over all spanning trees of $S$ of the number of incompatible chords which occur when the graph is marked as in the Correspondence Theorem.

Proof. From the definition of the line index, it is clear that this minimum is an upper bound. To establish that it is also a lower bound, we need to prove that in any signed graph there is a spanning tree which is disjoint from at least one minimum set of lines whose removal leads to balance. To do this it suffices to show that there is at least one such minimum line set whose removal does not disconnect the graph. Such a set is then a

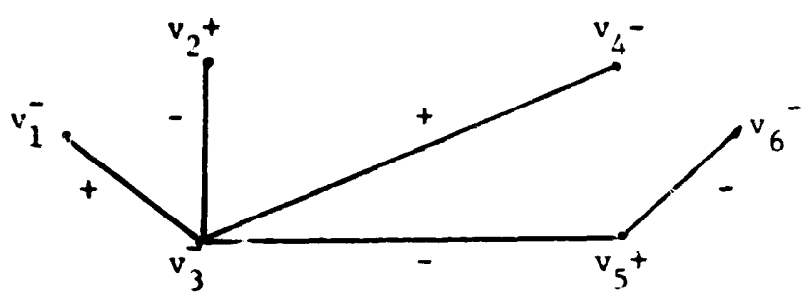

Fig. 3. 
collection of chords of some spanning tree. Suppose there is a signed graph for which this is not true and furthermore let $S$ be chosen so that the line index $k$ of $S$ is as small as possible. Clearly $k>1$, since no bridge can lie on a cycle. Now consider a smallest set of lines $E=\left\{e_{1}, e_{2}, \ldots, e_{k}\right\}$ whose removal results in balance, and take $S^{\prime}=S-e_{1}$. Certainly the line index of $S^{\prime}$ is $k-1$, so in $S^{\prime}$ there exists a minimum set of lines $E^{\prime}=\left\{e_{1}^{\prime}, e_{2}^{\prime}, \ldots, e_{k-1}^{\prime}\right\}$ whose removal changes $S^{\prime}$ to a balanced structure but does not disconnect it. In that case, however, the set $\vec{F}+e_{1}$ is a set of $k$ lines in $S$ whose removal yields balance but does not disconnect it. Thus contradiction establishes the theorem.

By this result, it becomes clear that a suitable modification of the balance algorithm will serve to calculate the line index of a given signed graph $S$, and at the same time identify a minimum line set whose deletion or negation balances $S$.

An additional bonus arising from the algorithm is an automatic division of the points of a balanced signed graph $S$ into coalitions. Simply note that the signs of the points of a negative line must differ while those of a positive line agree, so that the sets of negative points and positive points must be the two coalitions.

There are, of course, a host of additional problems which immediately suggest them. selves. First and foremost, we wish to obtain similar results for consistent marked graphs. Secondly, the connection between marked graphs and balanced signed graphs deserves to be more fully explored. In what ways are structural features of one reflected in the other?

\section{References}

A.V. Aho, J.E. Hopcroft and J.D. Ulman, The Design and Analysis of Computer Algorithms (AddisonWesley, Reading, 1974).

L.W. Beineke and F. Harary, Consistency in marked digraphs, J. Math. Psych. 18 (1978) 260-269.

L.W. Beineke and F. Harary, Consistent graphs with signed points, Rivista di Matematica per le Scienze Economiche e Sociale 1 (1978) 81-88.

D. Cartwright and F. Harary, Structural balance: A generalization of Heider's theory. Psych. Rev. 63 (1956) 277-293.

F. Harary, Graph Theory (Addison-Wesley, Reading, 1969).

F. Harary, On the measurement of structural balance, Behavioral Sci. 4 (1959) 316-323.

F. Harary, On the notion of balance of a signed graph, Michigan Math. J. 2 (1953-54) 143-146.

F. Harary and J.A. Kabell, Counting balanced sigued graphs using marked graphs, Proc. Edinburgh Math. Soc. Submitted.

F. Heider, Attitudes and cognitive organization, J. Psych. 21 (1946) 107-112. 